\title{
Analysis on the Impact of the Ritual of the Western Zhou Dynasty on the Bronze Ornamentation
}

\author{
Shuyan Pei \\ College of Historical Culture and Tourism \\ Xi'an University \\ Xi'an, China 710065
}

\begin{abstract}
The bronze ornamentation in the Shang Dynasty were mainly of imaginary animal pattern, changed into realistic animal pattern in the Western Zhou Dynasty, and simplified into geometric pattern until the middle and late Western Zhou Dynasty, which proved that the bronze ornamentation in the Western Zhou Dynasty has undergone fundamental changes. The reason of such changes lied in the change of social system by the rulers of the Western Zhou Dynasty, and thus resulted in the change of Zhou people's cognitive and aesthetic concepts. At present, there is no indepth research on the relationship between bronze ornamentation and cultural evolution in the academic circle, and the exploration on the reasons for the development and evolution of bronze ornamentation is relative less. Therefore, this paper analyzes on the impact of the ritual of the Western Zhou Dynasty on the bronze ornamentation from the research of bronze ornamentation.
\end{abstract}

Keywords-Western Zhou Dynasty; aesthetic; bronze ware; ornamentation

\section{INTRODUCTION}

The bronze ornamentation was originated from the ornamentation on the pottery wares that usually made by carving and colored drawing in the prehistoric era of China. With the continuous development of the Bronze Age, these primitive simple geometric patterns gradually evolved into animal pattern, with an abstract artistic style. A large number of animal patterns appeared on bronze wares illustrated the elevation of people's artistic aesthetic. All kinds of ornamentations on bronze wares were in combination with their marvelous shapes, created unique bronze culture with artistic quality of the Chinese Bronze Age.

\section{RESEARCH STATUES ON BRONZE ORNAMENTATION}

Previous studies on bronze ornamentation mostly focused on the study of ornamentation itself, including the naming, rubbing, painting, photography and other basic work, while in-depth studies mostly on ornamentation classification according to different patterns, or the dynastic research of similar ornamentation. Of course, the basic research on bronze ornamentation is of special importance, which has been mentioned in books of "Archaeological Map" and

CLC Number: K876.41 Document Identification Code: A Article Number:
"Bogu Map" written by people in the Song Dynasty, mainly described the naming of the ornamentation and the object it conveyed and made comments. The name of bronze ornamentation in the Qing Dynasty has been basically determined. Many names have been used up to now. However, due to the limited technology of the Qing Dynasty and the inaccuracy of hand-drawn designs, the original decoration cannot be truly displayed, and the artistic manner of beautifying ornamentation has been added.

It was not until 1941 that the naming of bronze decoration was standardized in Rong Geng's "Reference on the Bronze Wares in the Shang and Zhou Dynasties", in which the bronze ornamentation was systematically classified for the first time, the origin, continuity and innovation of each ornamentation and the different forms of expression of the same ornamentation type were studied, and the era of ornamentation was roughly explained. In addition to the classification and naming of ornamentation, there were also studies on the artistic achievements and technological level of patterns from the perspective of art and technology, mainly on the study of bronze ornamentation in the Yin and Eastern Zhou Periods. The research on the composition style, ornamentation theme, layout, drawing techniques, and making method and techniques can be regarded as the basic research of ornamentation itself.

In the early 1950s, Chen Mengjia also discussed ornamentation in "Dynastic Study on Bronze Wares in the Western Zhou Dynasty", summarized the characteristics of ornamentation on bronze and the law of its development and evolution, and proposed the shapes of bird patterns in different dynasties for the first time, classified and made dynastic research on the pattern. In addition to the study of ornamentation as the basis of dynastic study, there were also studies on a certain kind of ornamentation. Tan Danjiong's "New Chronology of Bronze Wares in the Spring and Autumn Periods and the Evolution of Dragon Pattern" was a paper that explored the development and evolution of dragon patterns from the Shang Dynasty to Warring States. Chen Gongrou and Zhang Changshou's "Dynastic Study on Bird Patterns on the Bronze Wares of the Yin and Zhou Dynasties" and "Dynastic Study on Animal Face Patterns on the Bronze Wares of the Yin and Zhou Dynasties", etc. were all research articles using ornamentation as the basis of dynastic study. The above researches have applied the 
development and evolution of bronze ornamentation in the dynastic study of bronze wares, elevated the research of bronze wares to a new height, and also reflected the importance of bronze ornamentation for the research on bronze wares.

The research in the cultural connotation conveyed by the ornamentation is of great importance that different ornamentation can reflect the ideology of the people and the political system at that time. On the contrary, the changes of people's ideology and social system also have a fundamental impact on the evolution of bronze ornamentation. Therefore, the study on the bronze ornamentation should not be limited to the classification and the dynastic study of bronze wares made according to the classification. The artistic value of bronze ornamentation cannot be neglected. However, the research on the connotation of bronze ornamentation is the weakest part, so it is very important to make a deep research on the art forms, ideology and social system.

\section{THE CHARACTERISTICS OF BRONZE}

\section{ORNAMENTATION OF ZHOU DYNASTY IN DIFFERENT STAGES}

The Shang culture was inherited in a certain degree at the early stage after the establishment of the Western Zhou Dynasty. From the quantity and form of the unearthed bronzes, the bronzes in the late Shang Dynasty (after the second stage of the Yin Ruins) entered a highly developed era. It can be seen from the results of excavation of bronze wares by the field archaeological units such as Zhou people's tombs and cellars that the bronze wares owned by Zhou people were not clear before the Emperor Wen. Until now, the earliest bronze wares of the Zhou Dynasty that unearthed were made during the reign of the Emperor Wen. At the early stage after the establishment of the Western Zhou Dynasty, the bronze manufacturing industry also lagged far behind the Shang Dynasty, and there was no great development in the shape of the objects. In addition, there was no obvious innovation in the ornamentation, mainly inherited the ornamentation of the Shang Dynasty, and the patterns were almost the same with those made in the Yin Ruins, which proved the backwardness of the Zhou Dynasty at the early stage. It is not difficult to see from the bronze ornaments in the early Zhou Dynasty and the early stage after the establishment of the Western Zhou Dynasty that the Zhou people inherited from the Yin people in the early stage on the formation of culture. Therefore, the formation of the Zhou culture was first inherited from the legacy of the ancestors, and gradually formed the bronze ornamentation with the style of the Zhou Dynasty with the constant changes and innovations, which has lasted throughout the Western Zhou Dynasty.

A large amount of realistic animal ornamentation appeared in the early Western Zhou Dynasty. Since the beginning of the Zhou Dynasty, the aesthetic style of Zhou people has gradually changed. At this time, the bronze ornamentation of Shang has not been abandoned, only some changes were made on the basis of the old model. From which we can see that although Zhou people inherited the old bronze ornamentation style of Shang in the early Western Zhou Dynasty, they also integrated their new knowledge and understanding. Especially in the late period of the early Western Zhou Dynasty, Zhou people's ideology and culture were more clearly reflected in the bronze ornamentation. "“The Reform by the Duke of Zhou' not only brought the establishment of ritual and music system, but also the changes in the aesthetic taste of the whole people. Since then, the bronze art of the Western Zhou Dynasty has a very different ornamentation style from that of the Shang Dynasty."[1] And gradually changed from the rich and complicated style of the Shang to the simple and plain style. Therefore, it can be regarded that the establishment of ritual system helped to set the concept of "respecting rites and making sacrifices while respecting ghosts and gods" [2].

Since the mid-Western Zhou Dynasty, the bronze ornamentation has undergone great changes, which can be regarded as the replacement of bronze ornamentation over its development that the original style of the Yin people gradually faded away and the new artistic style of Zhou people appeared in the bronze ornamentation. This process of gradual transformation basically went through the midWestern Zhou Dynasty. Until the late Western Zhou Dynasty, bronze ornamentation with the artistic style of the Zhou people has formed and the ornamentation with Shang style has no way to be found. Therefore, the Zhou people played a decisive role in the development of bronze ornamentation in the Western Zhou Dynasty. Under the influence of the cultural transformation of the Western Zhou Dynasty, the development of the Zhou people's bronze ornamentation underwent three transformation stages: the first was the stage of inheritance (early Western Zhou Dynasty), the second was the stage of evolution (mid-Western Zhou Dynasty), and the third was the stage of formation and development (late Western Zhou Dynasty).

From the historical and cultural development perspective, the period from King Wu of Zhou Dynasty to King Kang (early Western Zhou Dynasty) was a period from consolidation of political power to prosperity. This period was a process of gradual improvement and strengthening of political, economic and cultural aspects of Western Zhou Dynasty, and also a stage of gradual improvement of the ritual system of Zhou people. During the King Zhao and King $\mathrm{Mu}$ (middle of the Western Zhou Dynasty), ritual began to mature, characterized by following the principle of simplicity and plainness. There were great differences with Yin people in sacrifices especially that crops and plants were taken as the main sacrificial offerings instead of pigs, cow, sheep, dog and other animals. Feng Shengguo believed that "in the Zhou people's view, the success of man-made things depends on their own hands. There was no need to spend a lot of efforts to please ancestors, ghosts and gods. The motive of fascinating gods has been reduced, so that they did not have to spare efforts to kill cattle and sheep, or even use people as sacrificial offerings to please ancestors, ghosts and gods. In this way, the vegetables in March that can be seen everywhere were enough, which can explain why people in the Zhou Dynasty follow the principle of simplicity in sacrificial objects." [3] The simplicity and plainness in the ritual of Zhou people has become a noble value after a long period of development. Nowadays, the idea of advocating 
simplicity and opposing extravagance is still respected by people.

We believed that the transformation from animal pattern to geometric pattern during the mid-Western Zhou Dynasty was also related to the political system at that time. Geometric pattern began in the early stage of the midWestern Zhou Dynasty, during which the society was prosperous and stable, the political system was perfect, and the etiquette system was formed. The consciousness of advocating conciseness has been gradually deepened, people's perception of beauty has changed, so the ornamentation style of bronze ware has also changed. Although the new geometric ornamentation has not changed completely, nor can it be completely separated from the model matrix of animal pattern, the abstract concise feature embodied in the ornamentation has illustrated the understanding of beauty by Zhou people. During the period of King Zhao and $\mathrm{Mu}$, the national strength of the Western Zhou Dynasty became stronger and stronger, and the political focus has changed during the King of Gong, Yi, $\mathrm{Xiao}$ and $\mathrm{Yi}$ that they turned to stabilizing politics and strengthening internal construction instead of focusing on foreign expeditions and cruises. Therefore, Zhou people's characteristic of advocating simplicity and conciseness has become even more obvious that even bronze ornamentation highlighted this feature. It has completely separated from the style and characteristics of mainly using animal pattern, thus the geometric pattern with concise lines and orderly arrangement has been fully developed.

The reign of the Zhou Dynasty began to decline at the end of the Western Zhou Dynasty of King Li, and almost came to the end especially after the riots of the people. Bronze ornamentation did not develop greatly in this period. Although geometric pattern became popular, it only inherited the previous pattern and layout, and became more simplified, and new decoration did not appear. It can be seen that the development, evolution and replacement of ornamentation in the Western Zhou Dynasty were closely related to the ritual system and political environment of the Western Zhou Dynasty. On the contrary, it was under the political and ritual environment of the Zhou Dynasty that the concept and aesthetic style of the people changed, thus formed bronze ornamentation with the characteristics of the Zhou Dynasty.

\section{THE RELATIONSHIP BETWEEN AESTHETIC CONCEPT AND BRONZE ORNAMENTATION}

Bronze ornamentation has strong characteristics of the times. Most scholars believe that during the period from the Shang to Western Zhou Dynasty, bronze ornamentation was mostly imaginary animal patterns with mythological color, conveying a strong atmosphere of mystery and fear, which was inseparable from the harshness and cruelty of the rulers of the Shang Dynasty in the social system. Since the mid and late Western Zhou Dynasty, animal pattern gradually developed in a gentle direction, and almost disappeared in the late Western Zhou Dynasty. The bronze ornamentation was replaced by geometric pattern, which was inseparable from the ruler's placating political attitude and attaching importance to people's lives. By the middle and late Spring and Autumn Period, the ornamentation on bronzes was exquisite and elegant. The theme of the overall pattern was free and unrestrained, fresh and magnificent. Generally speaking, this evolution was the change of artistic style of the Shang and Zhou Dynasties, which also reflected the change of people's spiritual world and aesthetic concept. Therefore, the study of bronze ornamentation should not only take into account the artistic value displayed, but also to understand and explore the ancient social ideology through bronze ornamentation. According to the changes of the times, the ornamentation of different styles and characteristics were the exactly right materials to display the conditions of people's materials understanding toward the world and advocating of etiquette, which were of great value.

In the Western Zhou Dynasty, although the animal patterns on bronzes (excluding abstract or metamorphic animal patterns) did not last long, they began in the highly developed era of Chinese slavery society, and the conciseness and simplicity advocated by the later Zhou Dynasty gradually became a comprehensive social cognition. The Zhou people, who no longer worshipped animal sacrifices, completely changed the complex animal ornaments on bronzes and replaced them with simple and clear geometric ornaments with regular arrangement. The change of this phenomenon was not only an important change of ancient Chinese aesthetic concepts, but also a reflection of the change of social etiquette system in the Western Zhou Dynasty. People at that time have formed such concise and simple cognition, which has had a profound impact on Chinese civilization. On the contrary, under the influence of Zhou people's politics and etiquette system, bronze ornamentation has evolved or been replaced, and these changes in patterns were the powerful evidence of the changes in etiquette, ritual and law, social and cultural changes in the Western Zhou Dynasty.

\section{CONCLUSION}

In the ornamentation of bronze wares, the simplicity of Zhou people is particularly prominent. Zhou people paid more attention to the practicability of utensils, so the ornamentation of utensils was gradually simplified. From the layout of the ornamentation, it was transformed into ribbon ornamentation instead of ornamentation on the whole body of the utensils in the Shang Dynasty. And it tended to be simplified and regularized in shape and pattern. On the basis of the old animal patterns in Shang Dynasty, Zhou people merged the concise and abstract style and formed the concise geometric ornamentations characterized by neat arrangement. On the contrary, from the bronze wares unearthed in the middle and late Western Zhou Dynasty, geometric patterns have been widely used on bronze wares instead of the animal decoration of the Shang Dynasty. This was also in line with the simplicity of the Zhou people's ritual system, and the cultural characteristics of the Zhou people have obviously dominated the aesthetic standards of the people at that time, so they no longer use the complicated and gorgeous animal patterns as the aesthetic expression on bronze wares. It can be seen that the extensive use of this geometric pattern on bronze wares was also a strong proof of the change of 
aesthetic standards of Zhou people. Mr. Wu Zhenfeng once said, "the change of bronzes in the mid-Western Zhou Dynasty... basically abandoned the rule of bronze pattern on composition, changed into abstract pattern and adopted a large number of freer continuous composition methods... This change was a reaction of ideological change in bronze art.’[4]

To sum up, under the influence of the Shang and Zhou dynasties, the bronze ornamentation has been changing constantly, which has resulted in the change of people's aesthetic concepts. The changing law reflected by the ornamentation casted on bronze wares was also a true portrayal of the different ideas of the people in the Shang and Zhou Dynasties. While appreciating the artistic value of bronze ornamentation in the Shang and Zhou dynasties, people's worship and understanding of bronze ornamentation at that time should also be interpreted. We should explore its deep social value and significance from the changing bronze decorative patterns.

\section{REFERENCES}

[1] Jing Wen. Study on Realistic Animal Ornamentation of Bronze Wares in the Shang and Zhou Dynasties [D]. Master's Degree Thesis of the Northwestern University, 2006:42. (in Chinese)

[2] Zuo Qiuming. On Language, the Language of the Zhou Dynasty [M]. Shandong: Qilu Publishing House, 2005:202. (in Chinese)

[3] Feng Shengguo. The Simple and Concise Characteristics of the Rituals of Sacrifice in the Zhou Dynasty and its Reasons [J]. Lanzhou Academic Journal: 2013(9):32-36. (in Chinese)

[4] Editorial Committee of the Complete Collection of Chinese Bronze Wares. Complete Collection of Chinese Bronze Wares: Volume 5. [M]. Beijing: Cultural Relics Publishing House, 1996:26. (in Chinese) 